\title{
Enseñanza del algebra lineal en una facultad de ingeniería: Aspectos metodológicos y didácticos
}

\author{
Viviana Angélica Costa ${ }^{a}$ \& Raúl Rossignoli ${ }^{a, b}$ \\ ${ }^{a}$ Facultad de Ingeniería, Universidad Nacional de La Plata, La Plata, Argentina.vacosta@ing.unlp.edu.ar \\ ${ }^{b}$ Facultad de Ciencias Exactas, Universidad Nacional de La Plata, La Plata, Comisión de Investigaciones Científicas de la Provincia de Buenos Aires, \\ Argentina.raul.rossignoli@ing.unlp.edu.ar
}

\begin{abstract}
Resumen-En este trabajo describimos el enfoque con que se enseñan los conceptos relativos al Algebra Lineal en una asignatura del Área de Ciencias Básicas en una Facultad de Ingeniería de la República Argentina. Además exponemos los resultados de un cuestionario realizado a sus estudiantes que tiene el propósito de identificar las causas de los posibles obstáculos en la enseñanza y aprendizaje de esos conceptos, y que contiene también información sobre otros aspectos del curso. Se observa un acuerdo de los estudiantes con la metodología empleada, junto con algunas dificultades en el aprendizaje de ciertos temas y en comprender su vinculación con la Ingeniería. El mismo serviría de base para implementar estrategias didácticas o cambios metodológicos que reviertan las mismas, como así también el de brindar aportes que sirvan de referencia para dar iniciativa a posteriores investigaciones.
\end{abstract}

Palabras Clave - Algebra Lineal, educación matemática, ingeniería, metodología de enseñanza.

Recibido: 14 de septiembre de 2016. Revisado: 12 de octubre de 2016. Aceptado: 21 de octubre de 2016.

\section{Linear algebra teaching in a school of engineering: Methodological and didactic aspects}

Abstract - In this paper we describe the approach employed to teach the basic concepts related to linear algebra in a course of the Area of Basic Sciences in an Engineering Faculty of Argentina. Besides, we present the results of a questionnaire made for these students that aims to identify the origins of possible obstacles in the teaching and learning of these concepts, and which also contains information about other aspects of the course. An agreement of the students with the methodology employed is observed, together with some difficulties in the learning of particular topics and in understanding their connection with Engineering. This survey could be used to implement teaching strategies and//or methodological changes that may reverse the previous obstacles, as well as to provide input for further research initiatives.

Keywords - Linear Algebra, mathematics education, engineering, teaching methodology.

\section{Introducción}

En este trabajo nos enfocamos en la enseñanza del Álgebra Lineal en carreras de ingeniería. Los conceptos vinculados a esta rama de las matemáticas se estudian en los cursos básicos de los primeros años de los planes de estudio en esas carreras. Se estudian conceptos tales como vectores, matrices, sistemas de ecuaciones lineales, espacios vectoriales, transformaciones lineales, valores y vectores propios, y diagonalización de matrices. Estos conceptos, tienen conexión y utilidad para la resolución de diversos problemas en muchas áreas dentro de la matemática y también en otras ciencias e ingeniería. Por ejemplo, los sistemas de ecuaciones lineales, permiten modelar infinidad de problemas reales. Los valores y vectores propios asociados a una matriz o a una transformación lineal, poseen varias aplicaciones prácticas en la ingeniería. Entre ellas, podemos mencionar el estudio de la estabilidad de una estructura y sus frecuencias y modos naturales de vibración, el análisis de la convergencia de métodos numéricos para resolver sistemas de ecuaciones lineales algebraicas, la determinación de los ejes principales de inercia de un cuerpo rígido, la resolución de sistemas de ecuaciones diferenciales lineales y el estudio de la estabilidad de sistemas de ecuaciones diferenciales generales [1-3]. También tienen aplicación para el estudio de las formas bilineales y cuadráticas y aplicaciones en el Cálculo Infinitesimal como la optimización de funciones de varias variables. Además los conceptos del Álgebra Lineal son esenciales en la Teoría de Control Moderno [4].

En particular en la ingeniería aeronáutica, algunos conceptos del Álgebra Lineal son de importancia para la resolución de inestabilidades geométricas (pandeo), tensor de deformaciones en sólidos: direcciones principales de deformación, tensor velocidad de deformación en fluidos, inestabilidades aeroelásticas dinámicas ("flutter"), estabilidad dinámica de helicópteros, pararrotores y autogiros, diseño de sistemas de control, diseño de circuitos electrónicos, estabilidad dinámica longitudinal del avión (modos "fugoide" y "de periodo corto"), inestabilidades dinámicas laterales del avión ("rolido holandés" y otras), entre otras [5].

El aprendizaje del Álgebra Lineal es conocido como dificultoso. Algunas de las causas que se han identificado, se vinculan con su propia naturaleza epistemológica que generalmente presenta los objetos de estudio en forma abstracta $\mathrm{y}$ sin vinculación e interpretación física o geométrica, y con el uso de diferentes tipos de lenguajes [6].

Dada esta problemática, varios investigadores desde hace algunas décadas, han propuesto para una mejora en el aprendizaje, apartarse de la abstracción y acercarse a un primer curso de Algebra

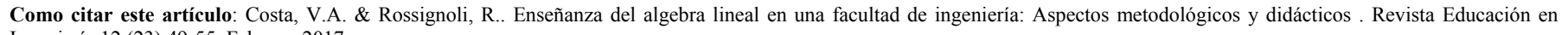
Ingeniería 12 (23) 49-55, Febrero, 2017. 
Lineal más concreto basado en matrices. Proponen enfocarse en el espacio $\mathrm{R}^{\mathrm{n}}$ en lugar de espacios vectoriales abstractos [7], enfatizando en su geométrica, considerando al producto vectormatriz A.x como una combinación lineal de las columnas de A y vinculando las ideas de espacio vectorial y transformaciones lineales con el estudio de sistemas lineales de ecuaciones. Por ello, se formó en 1990 el Linear Algebra Curriculum Study Group (LACSG), conformado por: David Carlson, Charles R. Johnson, David C. Lay y A. Duane Porter, y por otro lado, se conformó un grupo francés integrado por Jean Luc Dorier, Aline Robert, Jacqueline Robinet, Marc Rogalski, Michele Artigue, Marlene Alves Dias, Ghislaine Chartier, un grupo canadiense con Anna Sierpinska y Joel Hillel, y en EEUU Guershon Harel, Ed Dubinsky.

En la misma línea de trabajo en la Facultad de Ingeniería de la Universidad Nacional de La Plata, en la República Argentina (FIUNLP), un grupo de investigadores-profesores de una asignatura en la que se estudian los contenidos del Álgebra Lineal se encuentra desde hace una década interesados en mejorar su enseñanza y aprendizaje. Se busca que sus estudiantes logren adquirir el dominio conceptual y práctico de los objetos matemáticos involucrados de forma tal que les permita su correcta aplicación en la resolución de problemas en sus especialidades. Con ese propósito, se han desarrollado e implementado diversas estrategias didácticas y metodológicas [8], [9], con el objetivo de acercarse a las ideas que proponen los grupos de investigadores mencionados: un primer curso de Algebra Lineal cuyo plan de estudios y presentación respondan a las necesidades e intereses de las disciplinas de los estudiantes, orientado a matrices y que aliente el uso de la tecnología [10].

Esas estrategias implementadas han impactado en un mejor rendimiento académico de los estudiantes a lo largo de los últimos años y mayor participación y motivación en las clases. A pesar de ello, se observan dificultades en el aprendizaje de algunos conceptos.

Con el objetivo de trabajar a futuro sobre ello para revertirlas se instrumentó un cuestionario realizado a los estudiantes en el año 2015 y en 2016. Se exponen en este trabajo los resultados encontrados que además pueden servir de referencia a investigadores que actúan en los mismos escenarios de enseñanza.

\section{Marco institucional}

La investigación propuesta se lleva a cabo en la Facultad de Ingeniería (FI), una de las 17 que nuclea la Universidad Nacional de La Plata en la Provincia de Buenos Aires en la República Argentina. En la institución FI se dictan 12 carreras de ingeniería: Aeronáutica, Agrimensor, Civil, Computación, Mecánica, Electricista, Electromecánica, Electrónica, Industrial, Química, Hidráulica y Materiales. Los contenidos curriculares se distribuyen en 10 semestres y a su vez en Áreas: Ciclo Básico (CB), conformada por las asignaturas de Física, Matemática y Química, común a todas las especialidades (Tabla 1); el Área Tecnológica Básica (TB), el Área Tecnológica Aplicada (TA) y un Área complementaria (CO) específica para cada carrera.

Los cuatro primeros semestres, corresponden al CB, luego las asignaturas de TB y desde el séptimo semestre las asignaturas del área TA y CO.
Tabla 1

Asignaturas del Ciclo Básico.

\begin{tabular}{|c|c|c|}
\hline Año & Semestre & CB \\
\hline & $1^{\mathrm{o}}$ & $\begin{array}{l}\text { Matemática A: Cálculo diferencial en una y varias } \\
\text { variables. } \\
\text { Química General }\end{array}$ \\
\hline $1^{\circ}$ & $2^{\circ}$ & $\begin{array}{l}\text { Matemática B: Cálculo Integral en una y varias } \\
\text { variables. Cálculo Vectorial. Series numéricas. } \\
\text { Ecuaciones diferenciales ordinarias de primer orden. } \\
\text { Física I: Mecánica Clásica. } \\
\text { Química Inorgánica }\end{array}$ \\
\hline \multirow[t]{2}{*}{$2^{\circ}$} & $1^{\mathrm{o}}$ & $\begin{array}{l}\text { Matemática C: Series de potencias. Desarrollo de } \\
\text { Taylor. Algebra Lineal. Sistemas de ecuaciones } \\
\text { diferenciales lineales ordinarias. Temas de Cálculo } \\
\text { Numérico. } \\
\text { Probabilidades } \\
\text { Física II: Electricidad, Magnetismo y } \\
\text { Electromagnetismo. Ondas. } \\
\text { Química Orgánica I }\end{array}$ \\
\hline & $2^{\circ}$ & $\begin{array}{l}\text { Matemática D: Cálculo Avanzado } \\
\text { Estadística } \\
\text { Física III: Óptica } \\
\text { Química Orgánica II }\end{array}$ \\
\hline
\end{tabular}

Fuente: Los autores.

La asignatura sobre la que se realiza la investigación es Matemática $\mathrm{C}(\mathrm{MC})$ que es obligatoria para todas las carreras del CB. MC corresponde al $1^{\circ}$ semestre de $2^{\circ}$ año en todas las carreras excepto Ingeniería en Computación, que la ubican en el $2^{\circ}$ semestre de $2^{\circ}$ año, por lo que la mayor parte de los inscriptos del $2^{\circ}$ semestre corresponde a estudiantes que han vuelto a cursar ésta o alguna asignatura previa.

Se inscriben en promedio 550 estudiantes en el primer semestre, y entre 330 y 400 estudiantes en el segundo. Los estudiantes inscriptos se distribuyen en comisiones, con aproximadamente 60 a 70 alumnos según las carreras, a cargo de un equipo docente conformado por un profesor, un jefe de trabajos prácticos y dos auxiliares docentes (ayudante diplomado y ayudante estudiante), coordinados por el Profesor Titular. La carga horaria en MC es de nueve horas semanales, distribuidas en tres clases de tres horas cada una.

Los contenidos mínimos de la asignatura son: Series de potencias. Polinomio de Taylor y Serie de Taylor. Sistemas de ecuaciones lineales. Matrices, operaciones con matrices, matriz inversa, determinante, rango. Espacios Vectoriales, bases, dimensión. Números Complejos. Transformaciones Lineales. Valores y vectores propios de una transformación lineal. Diagonalización de matrices. Ecuaciones diferenciales ordinarias de segundo orden y sistemas lineales de ecuaciones diferenciales ordinarias. Álgebra Lineal Numérica: Matrices y operaciones relacionadas sobre un computador. Normas vectoriales y matriciales. Número de condición. Sistemas de ecuaciones lineales. Factorización de matrices: LU y Cholesky. Método de Jacobi y Gauss-Seidel. 


\section{Estrategias y recursos implementados}

El principal recurso educativo que los estudiantes utilizan para el estudio de los contenidos en MC es una Guía de Estudio [11] desarrollada por los profesores de la misma. En la Guía se desarrollan los contenidos distribuidos por temas. Cada uno comienza con una introducción que motiva y vincula los conceptos matemáticos con aplicaciones a la geometría, a la física, o a la ingeniería, luego se presentan los conceptos matemáticos teóricos, problemas resueltos, ejercitación para la práctica, preguntas para debatir, problemas para resolver utilizando software matemático y al final de cada capítulo se resumen los conceptos más importantes. La Guía de Estudio, constituye el eje central del aprendizaje, lo direcciona, lo estructura cronológicamente, delimita los contenidos mínimos, los saberes previos y además promueve un aprendizaje de tipo autónomo. También se sugiere la consulta de bibliografía seleccionada que sigue las recomendaciones dadas por el grupo Linear Algebra Curriculum Study Group [12-14].

El estudio de los contenidos en MC se distribuye en dos módulos de 18 clases cada uno, con una evaluación parcial para cada uno de ellos, de características teórico y práctico. El ambiente físico donde se desarrollan las clases tiene un "estilo de equipo" con mesas rectangulares y sillas dispuestas alrededor de cada una, que permite promover la interacción entre los estudiantes. Además por cada mesa, hay una computadora de escritorio disponible para su uso y una pequeña biblioteca con algunos de los libros de texto recomendados.

La metodología de trabajo en las clases tiene una modalidad llamada teórico-práctico, donde no hay espacios, ni horarios establecidos que separen las clases teóricas y las prácticas. Los docentes de cada equipo trabajan conjuntamente en el aula durante las tres horas de cada clase, distribuyendo el tiempo entre explicaciones en el pizarrón, consultas individuales o grupales, propuestas de problemas complementarios a los de la Guía, puestas en común para cerrar un tema y uso de software matemático como herramienta para la resolución de problemas. El profesor de cada equipo, es el que diseña y planifica las clases en acuerdo con las Guía de Estudio y del cronograma que aporta el profesor titular. Además, guía el trabajo de los estudiantes e interviene en las clases cuando así lo considera para generar espacios de discusión y de participación grupal, adaptándose a las necesidades que van surgiendo en el desarrollo de la clase y rediseñando las actividades si es necesario para permitir un uso eficiente del tiempo y de los recursos humanos. De este modo el profesor propiciaría un estilo de aprendizaje activo y centrado en el estudiante [15]. Además fuera de la clase los estudiantes disponen de un aula virtual en la plataforma educativa Moodle administrada por el mismo profesor en la que se presenta material educativo complementario al de la Guía de Estudio, un foro de novedades, un calendario, auto evaluaciones e información general de interés.

Los objetivos generales de $\mathrm{MC}$ son los de proporcionar al estudiante las habilidades algebraicas para resolver problemas que surjan en sus áreas de estudio y complementar el desarrollo analítico con los algoritmos numéricos. Establecer las conexiones entre los conceptos básicos de la teoría de espacios vectoriales y la teoría de sistemas de ecuaciones lineales. Aplicar la teoría de sistemas de ecuaciones lineales como modelo en la resolución de problemas, establecer las conexiones entre la teoría de matrices y la de transformaciones lineales e introducir los conceptos de valor y vector propio aplicados en la resolución de problemas.

Para la enseñanza de Espacios Vectoriales, se comienza estudiando en $\mathrm{R}^{2}$ y $\mathrm{R}^{3}$ tratando de recuperar conceptos $\mathrm{y}$ vincularlo con la geometría plana y en tres dimensiones, para luego pasar a $\mathrm{R}^{\mathrm{n}}$ en detalle y por último, a espacios más generales. Similarmente, se tratan las Transformaciones Lineales, dando importancia a la conceptualización y a las aplicaciones. Los Sistemas Lineales de Ecuaciones Diferenciales Ordinarias se estudian utilizando los conceptos de valores y vectores propios, y los de Espacio Vectorial y se complementa con diversas actividades de laboratorio con el objetivo de articular los conceptos matemáticos relativos a las ecuaciones ordinarias de segundo orden lineal con los conceptos del Movimiento Armónico Simple y Amortiguado estudiado en Física I [16].

La acreditación de MC se obtiene mediante: promoción directa o promoción por examen final. Para conseguir la aprobación por promoción directa se requiere que el alumno obtenga en cada evaluación parcial (son dos en total) una calificación mayor o igual a cuatro y que el promedio de las calificaciones obtenidas en dichas evaluaciones, sea mayor o igual que seis. Si un alumno no aprueba por promoción directa, pero obtiene una calificación mayor o igual a cuatro en los aspectos prácticos de todas las evaluaciones, obtendrá la aprobación de los Trabajos Prácticos y la habilitación para rendir el Examen Final de la asignatura. Además, con el fin de garantizar una evaluación uniforme en todos los grupos, los enunciados de los exámenes parciales son los mismos para todos.

El porcentaje de estudiantes, respecto del total de inscriptos durante 2015, que promocionó (y entre paréntesis, que aprobó o promocionó) fue de $60 \%(65 \%)$ en el primer semestre y del $51 \%(58 \%)$ en el segundo, pero los mismas ascienden a $87 \%$ (95\%) y 83\% (94\%) si se tiene en cuenta sólo aquellos estudiantes que no abandonaron el curso. La gran mayoría de los que abandonan no rinden ningún parcial. Los porcentajes de promocionados han ido aumentando en los últimos años, aunque la relación entre los semestres se mantiene.

\section{Metodología de la investigación}

La investigación realizada es un estudio de caso, del tipo descriptiva y su propósito es identificar las causas de los posibles obstáculos durante el proceso de enseñanza y aprendizaje de los conceptos del Algebra Lineal. Esto serviría de base para implementar cambios metodológicos o didácticos que permitan revertirlos, como así también el dar iniciativa a posteriores investigaciones.

Para ello se decide obtener datos a partir de la opinión de los estudiantes, utilizando un cuestionario como instrumento para la consulta, que se aplica a los que asisten a rendir el segundo módulo de MC en el primer semestre de 2015, en forma voluntaria y anónima. Se repite el experimento durante el año 2016, ante una mejora realizada en las Guías de Estudio para los temas de Sistemas de Ecuaciones Lineales, Matrices y Determinantes, 
Espacios Vectoriales y Transformaciones Lineales, a modo de conocer si hay cambios en las respuestas de los estudiantes respecto del año anterior.

El presente cuestionario se diseña con el fin de ampliar la información pública que obtiene periódicamente el Área Académica de FI UNLP como resultado de una serie de preguntas realizadas a todos los estudiantes, en forma obligatoria al finalizar los cursos, que indaga sobre algunas cuestiones académicas generales para cada materia. En la Tabla 2 se muestran las preguntas y sus resultados de 526 encuestados de 585 inscriptos en los cursos de MC en el primer semestre del 2015.

El cuestionario de esta investigación consta de cinco preguntas cerradas con algunas de selección múltiple. Los resultados obtenidos se cargan en tablas de modo de realizar un análisis cuantitativo, a partir de variables estadísticas.

Primero se pregunta sobre cuál es su carrera, con el objetivo de conocer el perfil de los estudiantes encuestados. Luego, se pregunta sobre su "asistencia a clase" pudiendo seleccionar una respuesta entre: "regularmente", "algunas veces", "casi nunca" o "nunca".

Tabla 2.

Resultados de las Encuestas para MC del Primer Semestre de 2015. ¿Cuántas horas cursó esta asignatura semanalmente en la facultad (en número)?

\begin{tabular}{lllll}
\hline $\mathrm{h}<3$ & $3 \leq \mathrm{h} \leq 5$ & $6 \leq \mathrm{h} \leq 8$ & $9 \leq \mathrm{h} \leq 12$ & $\mathrm{~h}>12$ \\
16 & 53 & 69 & 350 & 9 \\
\hline
\end{tabular}

¿Cuántas horas semanales, adicionales a las de cursada, le dedicó a las prácticas de la asignatura (en número)?

\begin{tabular}{lllll}
\hline $\mathrm{h}<3$ & $3 \leq \mathrm{h} \leq 5$ & $6 \leq \mathrm{h} \leq 8$ & $9 \leq \mathrm{h} \leq 12$ & $\mathrm{~h}>12$
\end{tabular}

\begin{tabular}{lllll}
88 & 189 & 108 & 73 & 25 \\
\hline
\end{tabular}

¿Cuántas horas semanales, adicionales a las de cursada, le dedicó al estudio de la asignatura (en número)?

\begin{tabular}{lllll}
\hline $\mathrm{h}<3$ & $3 \leq \mathrm{h} \leq 5$ & $6 \leq \mathrm{h} \leq 8$ & $9 \leq \mathrm{h} \leq 12$ & $\mathrm{~h}>12$ \\
154 & 182 & 67 & 51 & 28 \\
\hline
\end{tabular}

¿Cuántas veces se anotó para cursar esta materia?

\begin{tabular}{lll}
\hline $1 \mathrm{vez}$ & 2 veces & 3 o más veces \\
376 & 78 & 72
\end{tabular}

A su entender, ¿se desarrollaron en clase todos los contenidos de la asignatura? \begin{tabular}{lll}
\hline Sí & No & $\mathrm{Ns} / \mathrm{Nc}$ \\
\hline
\end{tabular}

$401 \quad 45 \quad 80$

¿Sintió que le faltó conocimiento previo para el cursado de esta asignatura?

\begin{tabular}{lll}
\hline Sí & No & $\mathrm{Ns} / \mathrm{Nc}$ \\
93 & 361 & 72 \\
\hline
\end{tabular}

¿Considera que los contenidos vistos en esta asignatura ya los había estudiado en otra (repetidos)?

\begin{tabular}{|c|c|c|c|c|c|}
\hline \multirow{2}{*}{\multicolumn{2}{|c|}{$\begin{array}{l}\text { Sí } \\
62\end{array}$}} & \multicolumn{2}{|l|}{ No } & \multicolumn{2}{|l|}{$\mathrm{Ns} / \mathrm{Nc}$} \\
\hline & & 386 & & 78 & \\
\hline \multicolumn{6}{|c|}{ ¿Cómo califica la planificación y organización global de la cátedra? } \\
\hline Excelente & $\begin{array}{l}\text { Muy } \\
\text { Bueno }\end{array}$ & Bueno & Regular & Malo & $\mathrm{Ns} / \mathrm{Nc}$ \\
\hline 92 & 167 & 160 & 30 & 11 & 66 \\
\hline \multicolumn{6}{|c|}{$\begin{array}{l}\text { ¿Cómo considera el nivel de exigencia de las evaluaciones parciales en relación } \\
\text { a los contenidos impartidos? }\end{array}$} \\
\hline $\begin{array}{l}\text { Alto } \\
59\end{array}$ & \multicolumn{2}{|c|}{ Razonable } & Bajo & \multicolumn{2}{|c|}{$\mathrm{Ns} / \mathrm{Nc}$} \\
\hline & \multicolumn{2}{|c|}{385} & 11 & \multicolumn{2}{|c|}{71} \\
\hline \multicolumn{6}{|c|}{ En función del nivel de exigencia, considera que la corrección fué: } \\
\hline \multirow{2}{*}{\multicolumn{2}{|c|}{$\begin{array}{l}\text { Acorde } \\
421\end{array}$}} & \multicolumn{2}{|l|}{ No Acorde } & \multicolumn{2}{|l|}{$\mathrm{Ns} / \mathrm{Nc}$} \\
\hline & & 25 & & 80 & \\
\hline \multicolumn{6}{|c|}{$\begin{array}{l}\text { ¿Cuál o cuáles de los siguientes medios complementarios de comunicación } \\
\text { utiliza la cátedra? }\end{array}$} \\
\hline Siu-Guaraní & $\begin{array}{l}\text { Web de la } \\
\text { Cátedra }\end{array}$ & Moodle & e-Mail & Otra & Ninguna \\
\hline 130 & 160 & 199 & 133 & 42 & 60 \\
\hline
\end{tabular}

Fuente: [17]
Tabla 3.

Pregunta 3.

\begin{tabular}{lc}
\multicolumn{1}{c}{ Aspecto } & $\begin{array}{c}\mathbf{1 ( m e n o r ) ~ a} \\
\mathbf{5 ( m a y o r )}\end{array}$ \\
\hline La organización/distribución de los temas durante el curso & \\
La Guía de Estudio & \\
La información en la página web de la cátedra & \\
Cantidad de horas de cursada & \\
Evaluaciones acordes a los contenidos &
\end{tabular}

Fuente: Los autores.

Tabla 4.

Pregunta 4.

\begin{tabular}{ll}
\hline \multicolumn{1}{c}{ Actividad dentro del aula } & Porcentaje \\
\hline Exposición del profesor & \\
Consultas e interacción con los docentes & \\
Trabajo en grupo con mis compañeros & \\
Trabajo individual & \\
Suma & $100 \%$ \\
\hline
\end{tabular}

Fuente: Los autores.

La tercera pregunta busca conocer la opinión de los estudiantes sobre algunos aspectos vinculados con la metodología, la organización de los cursos en MC, las Guías de Estudio, las evaluaciones y la cantidad de horas de cursada. Es de respuesta cerrada, y se les solicita que manifiesten su grado de acuerdo en una escala de 1 (menor) a 5 (mayor) (según la escala de Likert) para cada uno de los aspectos que se les presentan en una tabla, según consideran beneficiaron su aprendizaje (Tabla 3).

La cuarta pregunta busca investigar sobre las preferencias de los estudiantes acerca de la organización y distribución de las diversas tareas y actividades desarrolladas en las clases teórico-prácticas por el profesor y su equipo docente. Se les presenta una tabla donde se listan actividades áulicas y se solicita le asignen una parte porcentual de un total de $100 \%$ a cada una de ellas (Tabla 4).

Por último, la quinta pregunta indaga sobre la enseñanza y aprendizaje de los contenidos de la asignatura. Para ello se decide agruparlos en ocho temas: Series de Potencias y de Taylor (SPyT); Sistemas de Ecuaciones Lineales (SEL); Matrices, operaciones y determinante (MD); Espacios Vectoriales (EV); Transformaciones Lineales (TL); Valores y Vectores Propios, y Diagonalización (A); Ecuaciones Diferenciales (ED) y Cálculo Numérico $(\mathrm{CN})$, y para cada tema se presenta en una tabla de doble entrada una serie de afirmaciones:

- "Tuve muchas dificultades en su aprendizaje" (Df)

- "Le dediqué poco tiempo a su estudio" (Pt)

- "Comprendí el modo en que el profesor lo enseñó" (Cp)

- "Comprendí el modo en que está desarrollado en la Guía" $(\mathrm{Cg})$

- "Me faltaron conocimientos previos para su estudio" (Fc)

- "Es muy abstracto" (Ab)

- "Son muchos conceptos nuevos" (Cn)

- "Lo vinculé con la física y la ingeniería y le di un significado" (Fi)

- "Se estudió en poco tiempo" (Et) en la que se solicita a los estudiantes que seleccionen con un tilde las que consideren, pudiendo elegir más de una para cada tema. 


\section{Resultados y análisis}

El cuestionario fue respondido voluntariamente en forma anónima por 139 estudiantes durante el primer semestre del año 2015, y por 156 durante el primer semestre del año 2016, de entre 19 y 21 años de edad.

En la Fig. 1 se discrimina en porcentaje de cuáles carreras resultaron ser los estudiantes que respondieron el cuestionario. Predominando la cantidad de estudiantes de Ing. Química e Ing. Industrial, y en menor medida los estudiantes de las carreras Ing. en Materiales, Ing. Hidráulica e Ing. en Computación e Ing. Electricista que son las carreras con menor matrícula de ingreso.

En relación a la segunda pregunta, que indaga por la asistencia a las clases teórico-prácticas de carácter no obligatorio, se encontró que más del $75 \%$ de los estudiantes asisten regularmente, lo que revela la importancia que le conceden ellos a las mismas. El porcentaje de estudiantes que asistió "nunca" o "casi nunca" no supera el 10\% del total (Fig. 2).

En la Tabla 5 se muestran los resultados obtenidos. Para cada aspecto se expone el porcentaje de estudiantes que seleccionó tal valoración según la escala dada (1 menor - 5 mayor). Se observa que en relación a la "Organización/distribución de los temas durante el curso" el

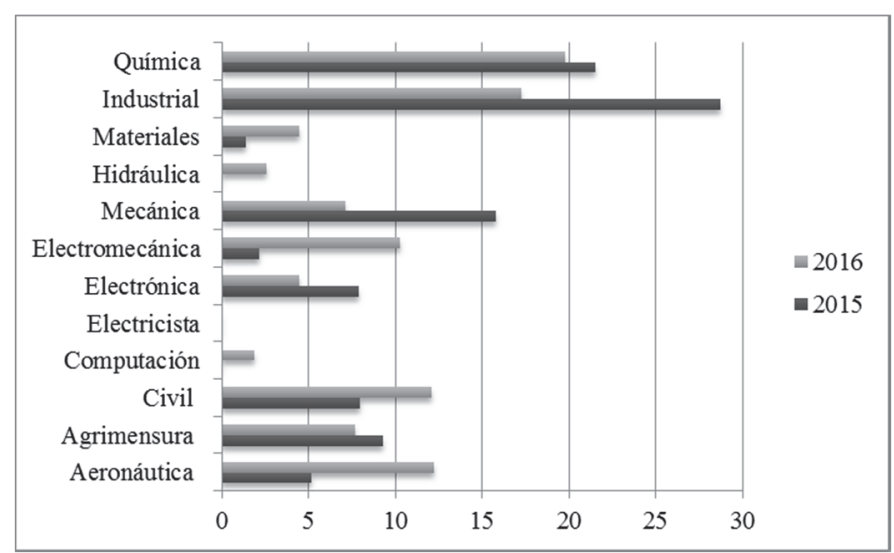

Figura 1. Porcentaje de estudiantes que responden el cuestionario según la carrera.

Fuente: Los autores

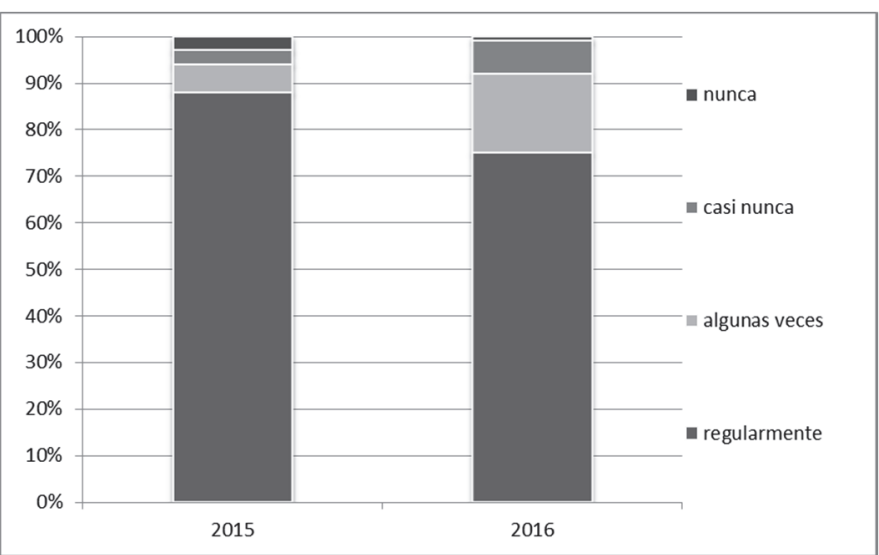

Figura 2. Asistencia a clase. Porcentajes. Años 2015 y 2016. Fuente: Los autores.
Tabla 5.

Porcentajes de valoración (1 menor - 5 mayor) para cada año.

\begin{tabular}{|c|c|c|c|c|c|}
\hline \multirow[t]{2}{*}{ Aspecto } & \multicolumn{5}{|c|}{$\begin{array}{c}\text { Escala } \\
\text { 1(menor) }-5 \text { (mayor) } \\
2015-2016\end{array}$} \\
\hline & 1 & 2 & 3 & 4 & 5 \\
\hline $\begin{array}{l}\text { Organización/distribución de } \\
\text { los temas durante el curso }\end{array}$ & $0-1$ & $4-7$ & $10-22$ & $44-36$ & $42-34$ \\
\hline La Guía de Estudio & $6-8$ & $21-18$ & $32-28$ & $28-31$ & $14-15$ \\
\hline $\begin{array}{l}\text { La información en la página } \\
\text { web de la cátedra }\end{array}$ & $22-12$ & $13-15$ & $15-27$ & $25-20$ & $24-27$ \\
\hline $\begin{array}{l}\text { Cantidad de horas de } \\
\text { cursada }\end{array}$ & $1-3$ & $4-4$ & $14-28$ & $22-22$ & $60-43$ \\
\hline $\begin{array}{c}\text { Evaluaciones acordes a los } \\
\text { contenidos }\end{array}$ & $1-1$ & $2-4$ & $9-10$ & $23-31$ & $65-54$ \\
\hline
\end{tabular}

Fuente: Los autores.

Tabla 6.

Porcentajes promedio que otorgan los estudiantes a cada una de las actividades en el aula.

\begin{tabular}{lrr}
\hline Actividad dentro del aula & $\mathbf{2 0 1 5}$ & $\mathbf{2 0 1 6}$ \\
\hline Exposición del profesor & 34 & 29 \\
Consultas e interacción con los docentes & 29 & 30 \\
Trabajo en grupo con mis compañeros & 21 & 20 \\
Trabajo individual & 16 & 21 \\
Total (\%) & 100 & 100 \\
\hline
\end{tabular}

Fuente: Los autores.

$86 \%$ lo valoran positivamente (escala 4 y 5 ) durante el año 2015 y $70 \%$ durante el 2016 . Esto es comparable con los resultados que se muestran en la Tabla 2, a la pregunta ¿Cómo califica la planificación y organización global de la cátedra? en la que el $79 \%$ la valora "Buena" o superior. Así mismo las "Evaluaciones acordes a los contenidos" es bien valorada (escala 4 y 5 ) por el $88 \%$ de los estudiantes durante el 2015 y por el $85 \%$ en el 2016 , en forma similar al $84 \%$ que se observa en la Tabla 2 para "Razonable" y "Alto" a la pregunta ¿Cómo considera el nivel de exigencia de las evaluaciones parciales en relación a los contenidos impartidos? En relación a la "Cantidad de horas de cursada" el $82 \%$ lo valoran positivamente (escala 4 y 5 ) en el 2015, mientras que durante el 2016 sólo el 67\%. En relación a la valoración asignada por los estudiantes al aspecto la "Guía de Estudio" se concentra entre las valoraciones 3 y 4 . Menos valorado es "La información en la página web de la cátedra".

En relación a la cuarta pregunta que indaga sobre la preferencia de los estudiantes en relación a la organización y distribución de las actividades vinculadas al proceso de estudio en una clase se encuentran los resultados que se muestran en la Tabla 6. Se observa que los estudiantes prefieren (en promedio) destinar cerca del $60 \%$ del tiempo total de una clase repartido entre las exposiciones del profesor y las consultas e interacción con los docentes, y el resto, entre el trabajo en grupo con sus compañeros y el trabajo individual.

En relación a la última pregunta, en la que se indaga sobre algunos aspectos en relación a la enseñanza y aprendizaje de los contenidos de $\mathrm{MC}$ se realizaron las siguientes consideraciones a los efectos de realizar un análisis y comparar el fenómeno de estudio entre los años 2015 y 2016. Dado que la cantidad de estudiantes 
Tabla 7.

Frecuencias marginales para cada tema.

\begin{tabular}{ccccccccc}
\hline \multicolumn{10}{c}{$\mathbf{2 0 1 5}-\mathbf{2 0 1 6}$} \\
& SPyT & SEL & MD & EV & TL & A & ED & CN \\
\hline Df & $10-20$ & $1-2$ & $1-4$ & $1-13$ & $10-17$ & $3-6$ & $10-10$ & $5-5$ \\
Pt & $7-10$ & $11-4$ & $9-7$ & $9-11$ & $11-11$ & $8-8$ & $8-7$ & $15-21$ \\
Cp & $20-21$ & $32-43$ & $31-36$ & $31-22$ & $23-21$ & $29-35$ & $23-29$ & $21-26$ \\
Cg & $10-12$ & $26-33$ & $26-32$ & $26-16$ & $15-17$ & $23-28$ & $15-23$ & $15-22$ \\
Fc & $4-6$ & $3-0$ & $3-2$ & $3-2$ & $6-2$ & $3-1$ & $4-3$ & $5-1$ \\
Ab & $12-13$ & $3-3$ & $7-6$ & $7-19$ & $12-12$ & $12-7$ & $7-6$ & $12-7$ \\
Cn & $10-4$ & $2-1$ & $7-5$ & $7-7$ & $11-8$ & $9-7$ & $6-8$ & $8-4$ \\
Fi & $20-2$ & $14-10$ & $11-4$ & $11-3$ & $6-3$ & $8-3$ & $19-8$ & $6-2$ \\
Et & $7-12$ & $7-4$ & $5-4$ & $5-7$ & $7-9$ & $5-5$ & $8-6$ & $13-12$ \\
Total (\%) & 100 & 100 & 100 & 100 & 100 & 100 & 100 & 100 \\
\hline
\end{tabular}

Fuente: Los autores.

que respondieron el cuestionario en ambos años no es la misma y además cada estudiante tenía la posibilidad de seleccionar más de una afirmación para cada tema, se decide organizar los resultados en una tabla de contingencia en las que se calcularon las frecuencias relativas marginales por temas (Tabla 7).

Se observa que para todos los temas, las afirmaciones "Comprendí el modo en que el profesor lo enseñó" (Cp) y "Comprendí el modo en que está desarrollado en la Guía" $(\mathrm{Cg})$ fueron las más seleccionadas por los estudiantes (presentan las mayores frecuencias). En particular para la afirmación $\mathrm{Cg}$, comparando los valores para el 2015 y el 2016, se observa un leve aumento en los porcentajes para los temas SEL, MD y TL. Esto indicaría que las mejoras realizadas en la Guía de Estudio para esos temas son positivas.

La afirmación "Tuve muchas dificultades en su aprendizaje" (Df) encuentra sus mayores porcentajes de selección para los temas SPyT, EV, TL y ED. Los temas SEL, MD y A son los que presentarían menos dificultades a los estudiantes. En relación a "Le dediqué poco tiempo a su estudio" (Pt) se observa que el tema $\mathrm{CN}$ es el más seleccionado. Esto podría deberse a que es el que se estudia al final del curso.

Para la afirmación "Me faltaron conocimientos previos para su estudio" las frecuencias observadas son muy bajas en ambos años, y además menores al 17\% que se observa en la Tabla 2, que contestaron "si" a la pregunta (del mismo tenor): ¿Sintió que le faltó conocimiento previo para el cursado de esta asignatura?

En referencia a la afirmación "Es muy abstracto", para ambos años se destacan los temas SPyT, EV, TL y ED. El tema TL es el más seleccionado en ambos años para la afirmación "Son muchos conceptos nuevos". Para la afirmación "Lo vinculé con la física y la ingeniería y le dí un significado", los temas de SPyT, SEL y ED fueron los más seleccionados durante 2015, y durante el 2016, los temas SEL y ED. Los temas CN, SPyT y TL son los más seleccionados en relación a "Se estudió en poco tiempo".

\section{Conclusiones}

En este trabajo se describieron aspectos metodológicos y didácticos con que se desarrollan los cursos en los que se estudian los conceptos del Álgebra Lineal en una Facultad de Ingeniería y se mencionó la importancia de los contenidos de esa rama de la Matemática para estudiantes en esas carreras como así también las dificultades que presentan su enseñanza y aprendizaje. Además se presentaron los resultados de un cuestionario realizado a los estudiantes que cursaron durante el primer semestre del 2015 y el primer semestre del 2016, que tenía como propósito identificar las causas de los posibles obstáculos durante el proceso de enseñanza y aprendizaje de los conceptos del Algebra Lineal. Por un lado, se obtuvo que los estudiantes concuerdan en varios aspectos metodológicos, didácticos y de organización de los cursos, como son las evaluaciones, la cantidad de horas de cátedra, la buena comprensión de la Guía de Estudio y de los profesores para el estudio de los temas. Estas cuestiones en conjunto, sumado al alto índice de asistencia a las clases, se reflejan en el alto rendimiento académico de los estudiantes, con un gran porcentaje de aprobación, que se conserva en los últimos años. También los estudiantes manifestaron acerca de las actividades en el aula, prefiriendo repartirlas entre explicaciones del profesor e interacción con sus compañeros y docentes del equipo, lo que indicaría una actitud activa de los estudiantes que es propiciada por el modo de enseñanza de estilo teóricopráctico que tienen los cursos de Matemática $\mathrm{C}$. En relación a la enseñanza y aprendizaje de los conceptos del Álgebra Lineal los estudiantes manifiestan que tuvieron dificultades con algunos temas, que algunos son "muy abstractos" y que necesitarían "más tiempo para su estudio", sobre todo para los temas de Series de Potencias y Taylor, Espacios Vectoriales y Transformaciones Lineales. Estas dificultades no se deberían a la falta de "conocimientos previos", sino que radicarían principalmente en que tienen "muchos conceptos nuevos". Por último cabe mencionar que si bien la Guía de Estudio presenta múltiples ejemplos y aplicaciones del Algebra Lineal a la Ingeniería y a la Física, igualmente los estudiantes muestran dificultades en vincular los conceptos estudiados con esas disciplinas, siendo este uno de los puntos a mejorar en el futuro.

\section{Referencias}

[1] Thomson, W., Theory of vibration with applications. New Jersey: Prentice Hall, 1993.

[2] Henry, R., Ecuaciones diferenciales: Una introducción moderna. Editorial: Reverte, 2008.

[3] Penney, D.E., Edwards, C.H., Ecuaciones diferenciales. Pearson Educación, 2008.

[4] Amato, F., Robust control of linear systems subject to uncertain timevarying parameters. Berlín: Springer, vol. 325, 2006.

[5] Costa, V.A., et al., Enseñanza del algebra lineal para alumnos de ingeniería aeronáutica: Experiencia motivadora, en Congreso Mundial y Exposición, Ingeniería 2010. Buenos Aires, 2010.

[6] Oktaç, A. y Trigueros, M., ¿Cómo se aprenden los conceptos de álgebra lineal?, Revista Latinoamericana de Investigación en Matemática Educativa, 13, pp. 4-11, 2010.

[7] Carlson, D., Johnson, C.R., Lay, D.C. and Porter, A.D., The linear algebra curriculum study group recommendations for the first course in linear algebra, The College Mathematics Journal, 24(1), pp. 41-46, 1993.

[8] Costa, V.A. y Vacchino, M.C., La enseñanza y aprendizaje del Álgebra lineal en la Facultad de Ingeniería, UNLP, en Congreso Chileno de Educación en Ingeniería, Chile, 2007.

[9] Costa, V.A. y Justo, C.E., El álgebra lineal en la resolución de problemas altimétricos de topografía, en Encuentro Educación Matemática en Carreras de Ingeniería, Argentina, 2015. 
Costa \& Rossignoli/ Revista Educación en Ingeniería 12 (23), pp.49-55. Febrero, 2017.

[10] Harel, G., The linear algebra curriculum study group recommendations: Moving beyond concept definition, MAA NOTES, pp. 107-126, 1997.

[11] Guía de Estudio. (2015). [Online]. Disponible: http://www.ing.unlp.edu.ar/catedras/F0304/

[12] Grossman, S.I., Algebra Lineal. México: MCGraw-Hill Interamericana, 2008.

[13] Poole, D., Álgebra lineal: Una introducción moderna. México D.F.: Cengage Learning, 2011.

[14] Lay, D.C., Algebra lineal y sus aplicaciones. México: Pearson Educación, 2007.

[15] Silberman, M., Aprendizaje activo: 101 estrategias para enseñar cualquier tema. México: Editorial Pax., 2005.

[16] Costa, V.A., Torroba P. y Devece. E., Articulación en la enseñanza en carreras de ingeniería: el movimiento armónico simple y las ecuaciones diferenciales de segundo orden lineal, Latin-American Journal of Physics Education, 7(3), septiembre, 2013.

[17] Encuestas de la Facultad de Ingeniería. (2015). [Online] Disponible: http://www.ing.unlp.edu.ar/sitio/encuestas/02sem15/default.php
V.A. Costa, recibió el título de Lic. en Matemática en 1989 por la Universidad Nacional de La Plata, Argentina, MSc. en Simulación Numérica y Control en 2002 por la Universidad Nacional de Buenos Aires, Argentina y el título de Dr. en Enseñanza de las Ciencias en 2013 por la Universidad Nacional del Centro de la Provincia de Buenos Aires, Argentina. Desde el año 1986 se ha desempeñado como docente en diversos cursos de Matemática en Facultades de la Universidad Nacional de La Plata (UNLP). Sus intereses investigativos incluyen: control de sistemas dinámicos y educación en ciencias. Actualmente es profesor con dedicación exclusiva en la Facultad de Ingeniería de la UNLP en la cátedra de Matemática C y Coordinador de la Unidad de Investigación, Desarrollo, Extensión y Transferencia: "Investigación en Metodologías Alternativas para la enseñanza de las Ciencias" (IMApEC).

ORCID: 0000-0003-1782-5378

R. Rossignoli, recibió el título de Lic. en Física en 1983 y el de Dr. en Física en 1987, ambos por la Universidad Nacional de la Plata, Argentina. Sus temas de investigación son: Mecánica Cuántica Teórica e Información Cuántica. Actualmente es profesor titular de Matemática $C$ en la Facultad de Ingeniería, profesor del Departamento de Física de la Facultad de Ciencias Exactas, ambas de la Universidad Nacional de La Plata, Argentina, e Investigador Científico de la Comisión de Investigaciones Científicas de la Provincia de Buenos Aires (CIC).

ORCID: 0000-0003-3827-2274 